\title{
50 Jahre Landesbibliothek im Neubau von Horst Linde
}

Vor 50 Jahren, am 3. August 1970, wurde das heutige Gebäude der Württembergischen Landesbibliothek an der Konrad-Adenauer-Straße in Stuttgart eröffnet. Anlass genug, um sich die Vorgeschichte, die Entstehung und die Architektur dieses Baus noch einmal genauer anzusehen.

\section{Vorgeschichte}

Die 1765 in Ludwigsburg vom württembergischen Herzog Carl Eugen gegründete Herzogliche Öffentliche Bibliothek war erst 1776 nach Stuttgart umgezogen. 1820 wurde die Bibliothek dann in das ehemalige Invalidenhaus verlegt, das direkt an der Neckarstraße, der heutigen Konrad-Adenauer-Straße lag. Weil dieses Gebäude aber - wie die vorherigen auch - unzureichend für eine Bibliothek war, erbaute man von 1878 bis 1886 ein eigenes Gebäude hinter dem ehemaligen Invalidenhaus, welches dann nach der Fertigstellung des neuen Bibliotheksgebäudes abgerissen wurde.

Dieses Gebäude von 1886 mit einem stattlichen, lang gestreckten Büchergebäude zur Straße hin und einem kleineren Verwaltungs- und Benutzungsgebäude, das sich dahinter anschloss, wurde im September 1944 fast vollständig zerstört, wobei insbesondere das Büchergebäude schwere Schäden erlitt und fast 500.000 Bände verloren gingen. In den folgenden Jahren wurden Teile des alten Gebäudes wieder notdürftig hergerichtet. Die Zukunft des Gebäudes blieb jedoch zunächst unklar. Im Juli 1951 wurde eine Baukommission gebildet, die ein Raumprogramm für eine neue Landesbibliothek erarbeitete. Zunächst war auch an die Unterbringung der Bibliothek in einen Flügel des wiederaufzubauenden Neuen Schlosses gedacht, der Stuttgarter Architekt Paul Bonatz (1877-1956) hatte hierzu schon einen Vorschlag ausgearbeitet. Auch der damalige Direktor der Bibliothek, Wilhelm Hoffmann (1901-1986), wünschte sich eine Unterbringung der Bibliothek im Neuen Schloss. Diese Gedanken zerschlugen sich aber letztendlich und auch der Wiederaufbau des gesamten alten Bibliotheksgebäudes wurde als zu teuer und zu wenig zweckmäßig angesehen.

\section{Pläne für einen Neubau}

Nachdem andere Alternativen verworfen worden waren, entschloss man sich zu einem Neubau der Bibliothek. Ein erster Vorschlag bezog sich auf das Grundstück der bisherigen Bibliothek, das sich ja bereits in Landesbesitz befand. Hierzu wurde 1954 wiederum von Paul Bonatz ein Vorschlag für einen Neubau auf den Grundmauern des alten Gebäudes gemacht. Der Nachteil: Man hätte den laufenden Betrieb der Bibliothek verlegen müssen. Eine zweite Idee sah einen Neubau auf dem Interimstheaterplatz vor, dort, wo heute das Landtagsgebäude steht.

Diese Lösung bot den Vorteil, ohne Überqueren der Konrad-Adenauer-Straße zur Bibliothek zu gelangen. Diese Möglichkeit schied jedoch aus, als 1956 der Neubau des Landtagsgebäudes auf diesem Gelände beschlossen wurde.

1958 und 1959 stellte man erste Beträge für vorbereitende Planungsarbeiten für einen Neubau der Landesbibliothek in den Landeshaushalt ein. Hier wurde auch festgelegt, dass das neue Bibliotheksgebäude und ein neues Gebäude für das Hauptstaatsarchiv als Baugruppe an der KonradAdenauer-Straße geplant werden sollten. Bereits ab 1958 liefen die Planungen für das Bibliotheksgebäude von drei Privatarchitekten unter der Leitung von Ministerialrat Dr. Horst Linde (1912-2016), dem Leiter der Hochbauabteilung im Finanzministerium Baden-Württemberg. Für diesen Bau wurde kein Architektenwettbewerb ausgeschrieben. Von Juli 1961 bis September 1963 baute man nun ein Interimsgebäude, das auch Teile des alten Büchergebäudes mitnutzte. Der Umzug der gesamten Bibliothek in dieses Interimsgebäude erfolgte im September und Oktober 1963, woraufhin das alte Verwaltungsgebäude abgerissen wurde. Das neue Gebäude sollte hinter dem Interimsgebäude entstehen, was den Vorteil hatte, dass dieses auch während der Bauarbeiten zunächst stehen bleiben und so der Bibliotheksbetrieb weiter aufrechterhalten werden konnte. Man wählte hier also die gleiche Vorgehensweise wie bei der Errichtung des Bibliotheksbaus von 1886. Aus diesem Grund 
liegt das Gebäude von 1970 auch sehr weit von der Konrad-Adenauer-Straße zurückgesetzt.

Die ersten Schätzungen der Baukosten beliefen sich auf 10 Mio. DM, 1963 schätzte man schon das Doppelte. Die Baupläne durchliefen im Jahr 1963 das Genehmigungsverfahren und am 29. Juni 1964 begann der Bau. Man hoffte, die Bauarbeiten in drei Jahren abschließen zu können. Bibliotheksdirektor Wilhelm Hoffmann hätte gern das 200-jährige Bibliotheksjubiläum am 11. Februar 1965 zum Anlass der Eröffnung des neuen Gebäudes genommen. Es war jedoch schnell klar, dass dieser Termin nicht eingehalten werden konnte. Am 5. Juli 1965 fand dann aber im Hauptstaatsarchiv die gemeinsame Grundsteinlegung für den so genannten „kulturellen Bereich" statt. Dazu gehörte die gesamte Kulturmeile, einschließlich Württembergischer Landesbibliothek, Musikhochschule und Staatsgalerie.

\section{Das Gebäude}

Die Baukosten inklusive der Inneneinrichtung betrugen am Ende annähernd 40 Millionen DM, viermal so viel, wie anfänglich geschätzt. Das fertige Gebäude hat eine Gesamtnutzungsfläche von 19.600 qm und umfasst einen umbauten Raum von 109.570 cbm. Es ist mit zwei Magazingeschossen unterkellert, deren Kapazität 2,4 Millionen Bände umfassen sollte. Mit fahrbaren Kompaktregalen war die Magazinkapazität auf 3,6 Millionen Bände erweiterbar. Man schätzte, dass die Kapazität bei einem jährlichen Zuwachs von 50.000 Bänden für 50 Jahre ausreichen würde und in der Tat wird nun nach genau 50 Jahren ein Erweiterungsbau in Betrieb genommen. Allerdings hat die Bibliothek schon seit

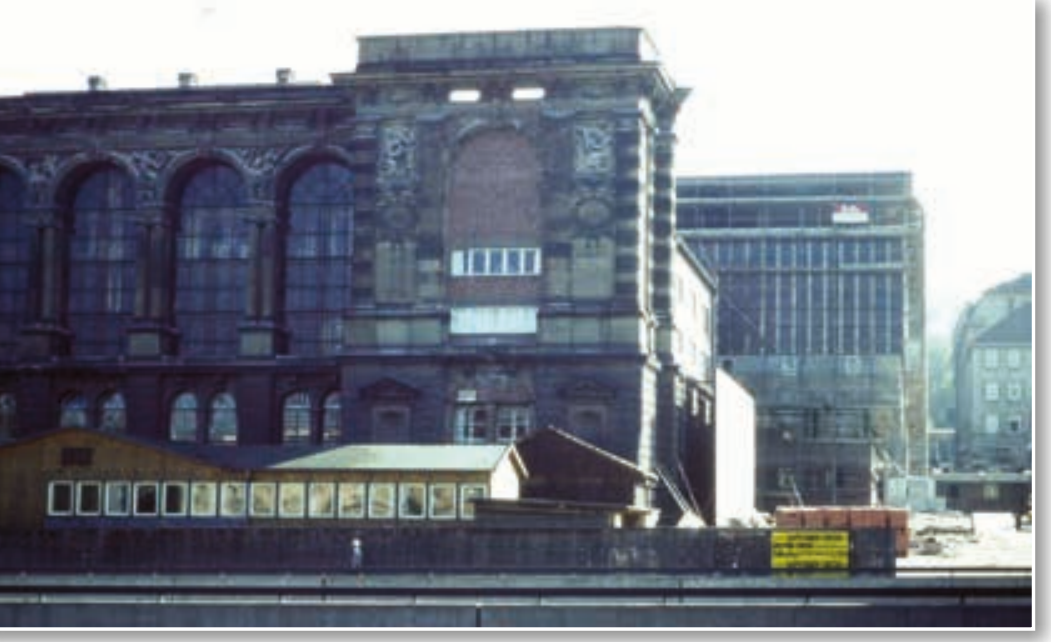

Abb. 1: Das Interimsgebäude der WLB, im Hintergrund sieht man einen Teil des Lesesaaltraktes des neuen Gebäudes
Jahrzehnten Ausweichflächen: ein Außenmagazin in Fellbach seit 1991 und zwei Geschosse in einem Bürogebäude in der Gaisburgstraße in Stuttgart seit 1993, wo zwei der Sondersammlungen mit ihren Beständen untergebracht sind. In das Magazin wurde mit einer Rohrpostanlage und einem mechanischen Transportband moderne Kommunikationsund Transporttechnik eingebaut, die heute jedoch eher Museumswert hat.

Das Gebäude ist klar gegliedert in drei bzw. vier Gebäudeteile: einen Verwaltungstrakt, einen Benutzungstrakt, einen Lesesaaltrakt und - wenn auch unter der Erde und damit von außen nicht sichtbar - einen Magazintrakt. Damit spiegeln die Gebäudeteile deutlich die unterschiedlichen Funktionen einer

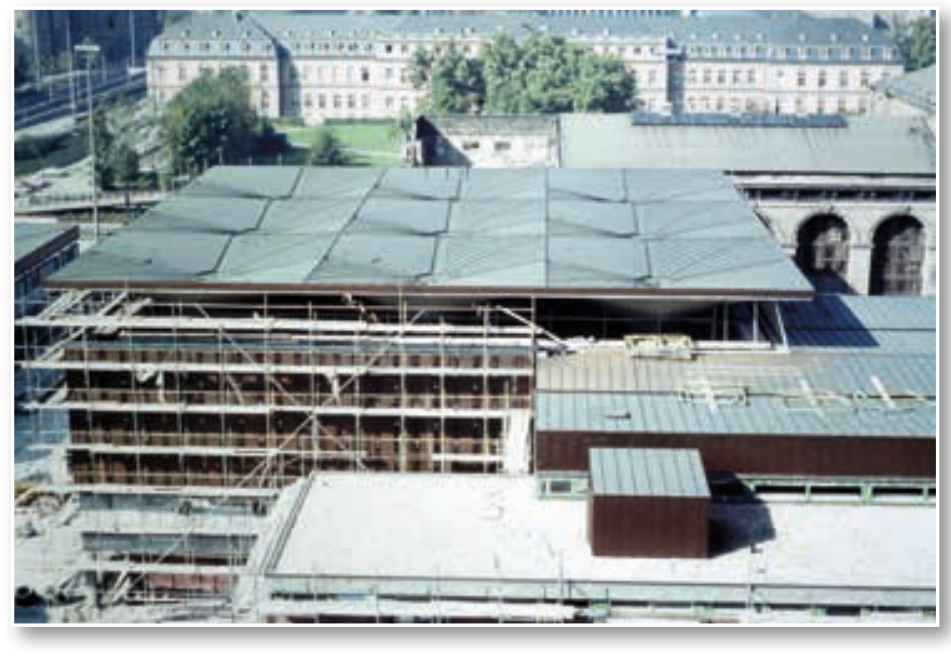

Abb. 2: Das neue Bibliotheksgebäude im Bau

Bibliothek wider. Seit dem 19. Jahrhundert waren zahlreiche Bibliotheksbauten nach diesem Schema gegliedert.

Der Lesesaaltrakt beherbergte verschiedene Katalogräume und Sonderlesesäle. Neben dem Hauptlesesaal, der auf 50.000 Bände ausgelegt war, gab es anfänglich einen Bibliographien-Lesesaal mit 20.000 Bänden, einen Handschriften-Benutzungsraum mit einer Handbibliothek von 10.000 Bänden, einen Musiklesesaal mit 6.000 Bänden, einen Benutzungsraum für die Graphischen Sammlungen und die Kartensammlung, mehrere Archive, 18 „Forscherzellen", Schreibmaschinenräume, Abhörkabinen sowie einen Lesesaal für aktuelle Literatur. Insgesamt bot die Bibliothek den Benutzern so 650 Arbeitsplätze und 100.000 frei zugängliche Bände als Präsenzbestand. Der Konzeption der Bibliothek lag der Gedanke zugrunde, „einer möglichst großen 
Zahl differenzierter Benutzergruppen eine möglichst große Zahl wissenschaftlicher und bibliographischer Literatur zum unmittelbaren Gebrauch zur Verfügung zu stellen", wie Wilhelm Hoffmann 1971 schrieb.

Im Publikumsbereich befindet sich ebenerdig die Garderobe. Die Bibliothek war von insgesamt drei Eingängen zugänglich. Über eine große repräsentative Treppe, wie in vielen Bibliotheksgebäuden, insbesondere des 19. Jahrhunderts, typisch, gelangt man auf die Hauptebene. Hier waren vormals die (Zettel-)Kataloge mit ihren großen Katalogschränken untergebracht, heute befindet sich an dieser Stelle der Selbstabholerbereich. Auf der Empore - noch eine Treppe höher - war beim Einzug der Bibliothek der Zentralkatalog Baden-Württemberg untergebracht. Der Verwaltungstrakt beherbergt bis heute ausschließlich Büro- und Besprechungsräume verschiedener Größe. Im Untergeschoss war das Buchmuseum mit großen Glasvitrinen eingerichtet, das lichtgeschützt in einem unteren Bereich des Gebäudes eingebaut war und Raum für Dauer- oder Wechselausstellungen bot. Ein Vortragsraum fehlte dem ursprünglichen Bau bei seiner Einweihung 1970 noch, war jedoch schon geplant. Er sollte vor dem Bibliotheksgebäude über der dortigen Tiefgarage entstehen, vorerst wurde jedoch nur

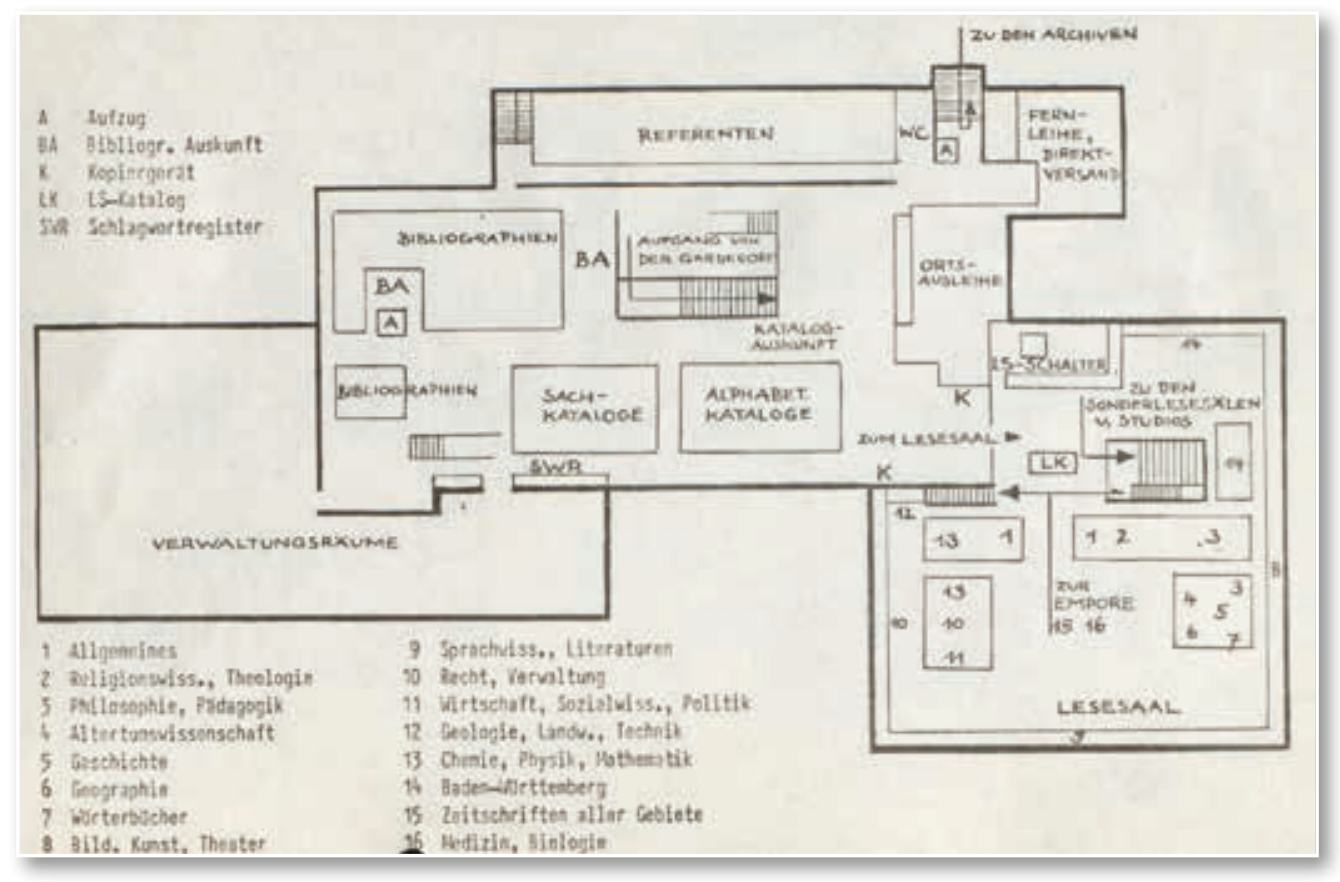

Abb. 3: Plan der Bibliothek, aus einem Bibliotheksführer von 1971. Deutlich zu sehen ist die Dreiteilung des Gebäudes.

worden vom Berliner Landschaftsarchitekten Walter Rossow (1910-1992).

\section{Architektonische Besonderheiten des Gebäudes}

Der Verwaltungstrakt steht in Richtung Ulrichstraße, an inn schließen sich die Publikumsräume entlang der Urbanstraße an. In Richtung Hauptstaatsarchiv liegt dann der Lesesaaltrakt. Dazu kam der unter den Lesesälen eingeschobene Flachbau der ehemaligen Bibliotheksschule sowie die Hausmeisterwohnung in einem separaten Bau, der ursprünglich ebenfalls Teile der Bibliotheksschule enthielt.

Zwischen den Publikumsräumen und dem Verwaltungstrakt bildete die Cafeteria einen Übergang. Das heutige Gebäude präsentiert sich als Stahlbetonskelett-Konstruktion, die mit Sichtmauerwerk ausgefacht wurde. Beton und Sichtmauerwerk sind überall ohne Verkleidung. Ursprünglich waren überall Holzfenster eingebaut worden, diese sind teilweise durch Kunststofffenster ersetzt worden, unter Beibehaltung des grün-blauen Farbkonzepts. Die verschiedenen Baukörper haben alle einfache Flachdächer, die mit Kupfer verkleidet sind. Das Obergeschoss kragt aus und steht auf Betonstützen. Dominant sind überall die Materialien Beton und Sichtmauerwerk, die nicht verkleidet sind.

Im Lesesaaltrakt ist das Dach des Hauptlesesaals das herausragende gestalterische Element, das aus insgesamt neun "Pilzen" besteht, die sich aus je vier HP-Schalen (Hyperbolische Paraboloidschalen) von je acht Zentimeter Dicke zusammensetzen. Die Stützen dienen gleichzeitig der Entwässerung. Die hohe Beton-Fassade des Lesesaals ist in regelmäßigen Abständen horizontal von schmadie Tiefgarage errichtet. 1971 wurde das Gebäude offiziell eingeweiht, die gesamten Außenanlagen waren im Sommer 1973 fertig. Sie waren geplant len Glaselementen durchbrochen. Die Betonteile der Fassade sind außen mit brettschichtverleimten Holzelementen verkleidet, die von Kupferblech um- 
geben sind. An der Innenseite sind jeweils schräg gestellte Holzelemente angebracht, die eine indirekte Beleuchtung des Innenraums über die Glaselemente der Außenwand ermöglichen, gleichzeitig aber den direkten Blick nach außen verhindern. Bei passendem Lichteinfall kann man die hinter den Glaselementen liegenden inneren Holzelemente gut erkennen.

Die beiden unterirdischen Magazingeschosse wurden in eine wasserdichte Kupferwanne eingebaut. Sie nehmen eine Fläche von jeweils $120 \times 55$ m ein und sind in sieben Brandabschnitte unterteilt.

\section{Das Gebäude im Gebrauch}

Nach der Fertigstellung des Gebäudes zog die Bibliothek vom Interimsgebäude in den Neubau ein; dazu diente auch eine Brücke, die vom alten in das neue Gebäude geschlagen worden war.

Am 3. August 1970 wurde das Gebäude - wie schon erwähnt - offiziell eröffnet. Im Oktober begann dann der Abbruch der Reste des alten Gebäudes bzw. des Interimsgebäudes. Dieser zog sich bis zum Februar 1971 hin.

Das zunächst großzügig dimensionierte und klar gegliederte neue Gebäude brachte im Lauf der Zeit auch einige Nachteile mit sich. Der offene Charakter des Mitteltraktes hatte den Nachteil, dass Gerüche aus der Cafeteria durchs ganze Haus strömten und dass sich die warme Luft am höchsten Punkt des Gebäudes sammelte. Auch der Schall breitete sich über sämtliche Geschosse aus. Schwierig war auch der offene Ausstellungsbereich, was klimatische, insbesondere aber Probleme bei der Überwachung von Ausstellungen mit sich brachte. Aus heutiger Sicht war auch die Einrichtung vieler verschiedener Speziallesesäle von Nachteil, da sie einen extrem hohen Personalaufwand nach sich zog.

Im Innern erfolgten im Laufe der Jahre auch einige größere Veränderungen. 1989 wurde eine zweite Leihstelle im Untergeschoss eingerichtet, 2003 die Theke der oberen Leihstelle erneuert. Im gleichen Jahr ging man ein weiteres großes Problem des Gebäudes an: An verschiedenen Stellen im Haus war Asbest verbaut worden. Zuerst saniert wur- de 2003 und 2004 der Boden im Lesesaal, später folgte ein Abschnitt im Magazin. Dort ist auf der gesamten Fläche asbesthaltiger Estrich verbaut worden. Die Sanierung dieser zwei fußballfeldgroßen Flächen soll in den nächsten Jahren in Angriff genommen werden.

2004 baute man die alten Zettelkataloge auf der Katalogebene ab (diese waren zuvor in die digitale Form überführt worden), und auf dem frei gewordenen Platz wurde der Selbstabholerbereich eingerichtet. Der Blick in den modern gestalteten Bereich mit verschiedenfarbigen Stirnwänden der Regale fungierte fortan als eine Art Erkennungszeichen für die Bibliothek und fand sich sowohl auf der Home-

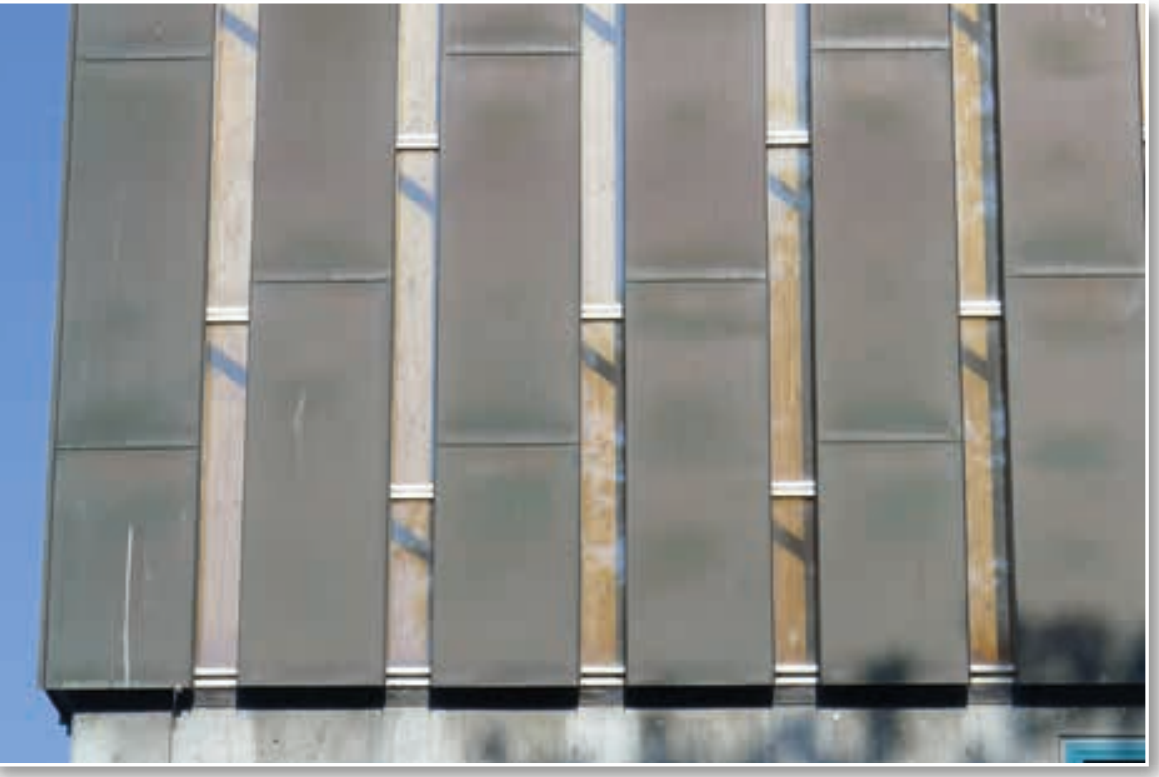

Abb. 4: Architektonische Details der Lesesaalfassade von außen

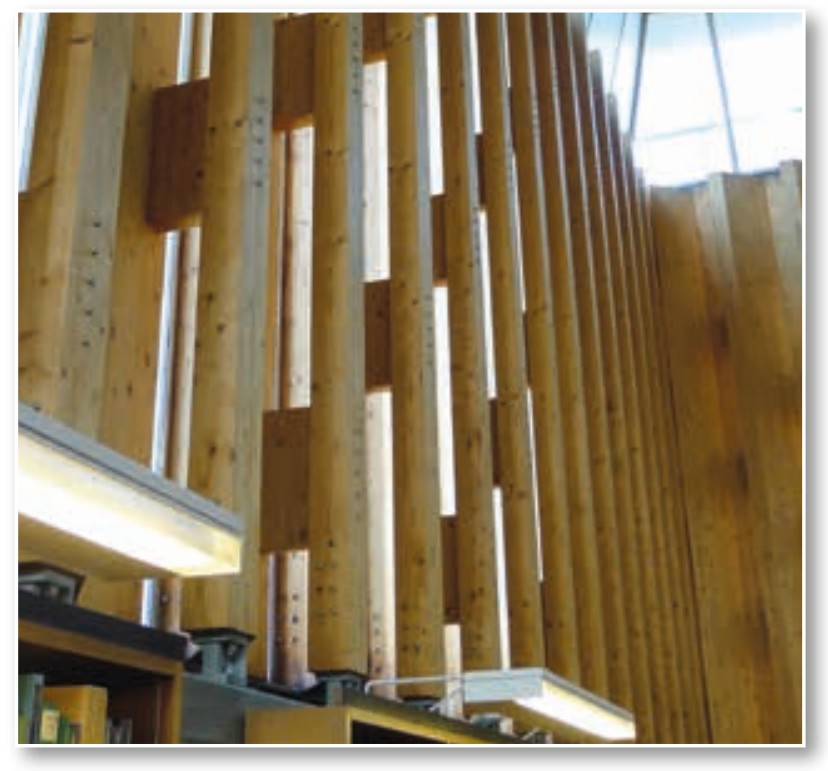

Abb. 5: Architektonische Details der Lesesaalfassade von innen 
page der Bibliothek wie auch auf eigens gedruckten Lesezeichen, die an die Nutzerinnen und Nutzer abgegeben wurden.

Problematisch war und ist die fehlende Barrierefreiheit des Gebäudes, das von zahlreichen Treppen und Treppchen und zum Teil sehr engen Fahrstühlen geprägt ist. Die an sich attraktiven großen Fensterfronten haben den Nachteil, dass sie sehr

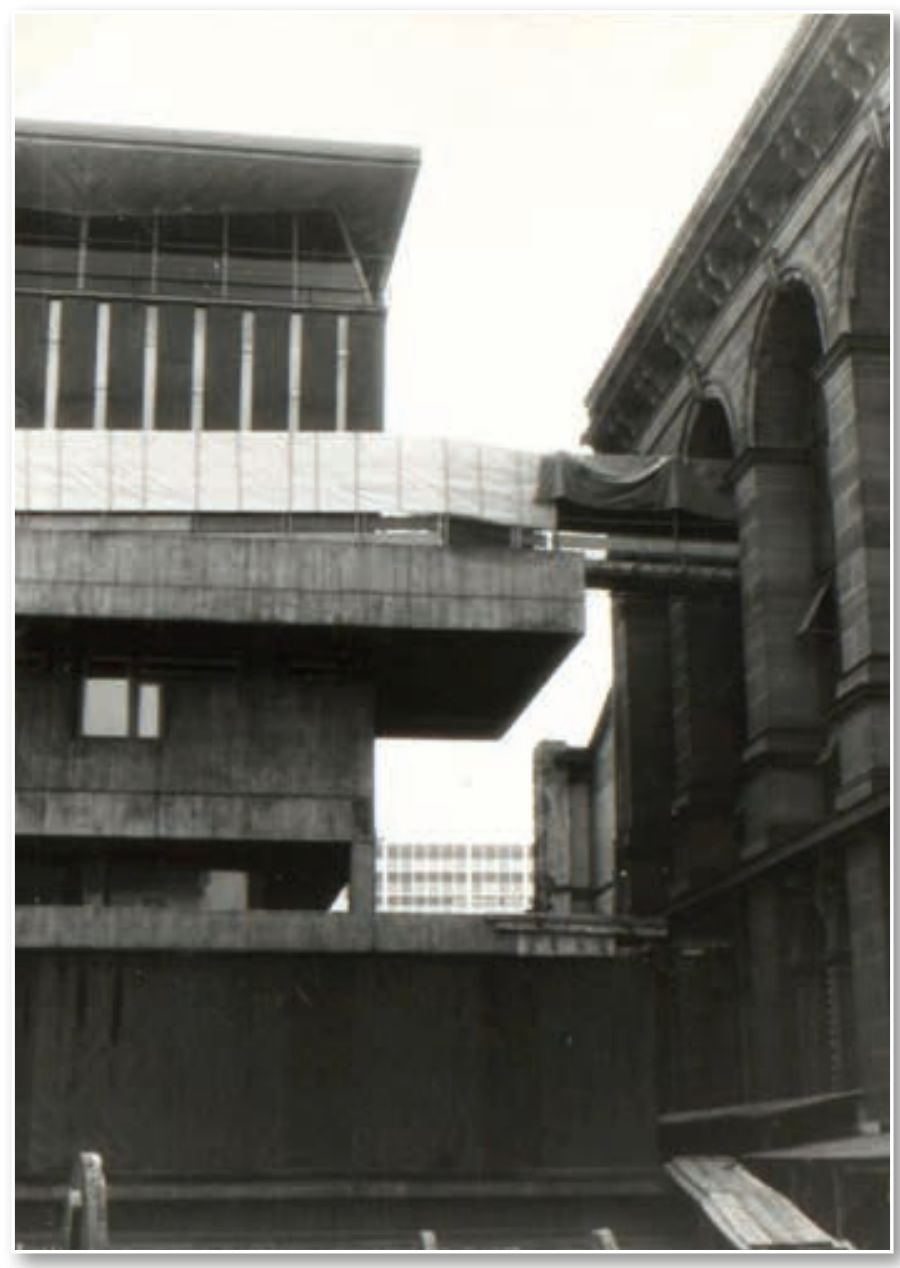

Abb. 6: Brücke vom Interimsgebäude in das neue Gebäude beim Umzug

schwierig zu reinigen und energetisch problematisch sind. 2007 konnten immerhin die meisten Fenster durch moderne, besser isolierte ausgetauscht werden.

Der größte Nachteil ist jedoch sicherlich die Grundkonzeption als - praktisch reine - Magazinbibliothek, eine heute absolut unzeitgemäße Organisationsform. Außerdem sind die Magazine schon seit Jahren zu klein, was eine Auslagerung verschiedener Bereiche nötig machte. Es rächte sich sehr schnell, dass der Entwurf für die Magazine auf dem Grundstück der Bibliothek keine Erweiterungsflächen vorgesehen hatte. Dies gehörte eigentlich seit dem 19. Jahrhundert zu einer der Grundanforderungen an Bibliotheksgebäude. Was dem Bau daneben heute jedoch besonders fehlt, sind große Freihandbereiche und eine wesentlich größere Anzahl an Benutzer-Arbeitsplätzen. Dieses Manko soll der geplante Erweiterungsbau beheben und auch sämtliche Außenstellen sollen wieder in das Hauptgebäude zurückgeholt werden, wenn das Gebäude von 1970 voraussichtlich im Jahr 2025 saniert worden sein wird.

\section{Hans-Christian Pust}

Literatur:

- Brodbeck, Walter / Schlumberger, Hans-Jörg: Bauaufnahme Württembergische Landesbibliothek 1750-1983, Stuttgart: Institut für Baugeschichte und Bauaufnahme, Stuttgart 1983.

- Hoffmann, Wilhelm: Stuttgart, Württembergische Landesbibliothek. In: Totok, Wilhelm / Weimann, Karl-Heinz (Hg): Regionalbibliotheken in der Bundesrepublik Deutschland, Frankfurt a.M.: Klostermann 1971 (Zeitschrift für Bibliothekswesen und Bibliographie, Sonderheft; 11), S. 254-263.

- DFW: Dokumentation, Information, Jg. 11 (1962/63), Heft 4, S. 156-157.

- DFW: Dokumentation, Information, Jg. 16 (1967/68), Heft 2, S. 39.

- Baumeister 1971, Nr. 7, S. 853.

- Detail, 1972, Heft 3, S. 467-470.

- Die Bauverwaltung, Jg. 45, 1972, Heft 5, Mai 1972, S. 222-227.

- DFW: Dokumentation, Information, Sonderheft Bibliothekskongress 1978.

- DBZ: Deutsche Bauzeitschrift, 1972, Heft 11, S. 2195-2198.

- Gerhard Liebers u.a. (Hg.): Bibliotheksneubauten in der Bundesrepublik Deutschland, Frankfurt / M.: Klostermann 1968 (Zeitschrift für Bibliothekswesen und Bibliographie, Sonderheft; 9), S. 306-313.

Abbildungsnachweis:

Abb. 1, 2, 3, 6: WLB

Abb. 4, 5: Hans-Christian Pust 